\title{
REFLEXIONES SOBRE EL BIENESTAR UNIVERSITARIO VIRTUAL EN COLOMBIA
}

\section{REFLECTIONS ON COLLEGE VIRTUAL COUNSELING IN COLOMBIA}

Germán Andrés Torres Escobar german.torreses@amigo.edu.co Universidad Católica Luis Amigó Bogotá-Colombia

Laura Giraldo Roncancio laura.giraldoro@amigo.edu.co Universidad Católica Luis Amigó Bogotá-Colombia

Paola Andrea Restrepo Benavides paola.restreposa@amigo.edu.co Universidad Católica Luis Amigó Bogotá-Colombia

Angie Katherine Giraldo Benavides leidy.giraldobe@amigo.edu.co Universidad Católica Luis Amigó Bogotá-Colombia

Leidy Dayhana Giraldo Benavides leidy.giraldobe@amigo.edu.co Universidad Católica Luis Amigó Bogotá-Colombia

Diana Catalina Montenegro Cedeño diana.montenegroce@amigo.edu.co

Universidad Católica Luis Amigó Bogotá-Colombia

Erenia Chavarro Ramos erenia.chavarrora@amigo.edu.co Universidad Católica Luis Amigó Bogotá-Colombia

Blanca Irene Villegas Soto blanca.villegasso@amigo.edu.co Universidad Católica Luis Amigó Manizales-Colombia 


\section{RESUMEN}

El presente artículo tiene como objetivo describir los servicios en salud mental que se ofertan desde el área de bienestar universitario en Colombia, así como, los alcances y limitaciones, entre ellos, la falta de una distinción clara en la ley entre servicios en salud física y mental y la baja cobertura de los servicios en salud mental para toda la comunidad estudiantil. Frente a estas dos falencias se plantea una propuesta para mejorar la oferta de servicios en salud mental que incluye aspectos como: la implementación de un servicio de consejería psicológica virtual y el aumento en el número de profesionales que atienden a los estudiantes universitarios. Finalmente, se mencionan los retos que existen para lograr la implementación adecuada de esta propuesta en el futuro como son: la necesidad de formar profesionales en consejería psicológica virtual e incluir en la ley del psicólogo colombiano las características de los servicios psicológicos virtuales y los lineamientos que se deben considerar para ofertar servicios de consejería psicológica virtual de buena calidad.

Palabras clave: bienestar universitario, educación virtual y a distancia, educación superior, consejería psicológica virtual, salud mental.

\section{ABSTRACT}

The present article aims to describe the mental health services that are offered from the university welfare area in Colombia, as well as, its scope and limitations, such as the lack of a clear distinction in the law between physical and mental health services and, the low coverage of mental health services for the entire student community. In order to deal with these two shortcomings, a plan is proposed to improve the offer of mental health services that includes aspects such as the implementation of a virtual psychological counseling service and the increase in the number of professionals who attend university students. Finally, the challenges that exist to achieve the adequate implementation of this proposal in the future are mentioned, among them, the need to train professionals in virtual psychological counseling and the inclusion in the Colombian psychologist's law of the characteristics of virtual psychological services and the guidelines that must be considered to offer good quality virtual psychological counseling services.

Keywords: university welfare, virtual and distance education, higher education, psychological counseling, virtual psychological counseling, mental health. 


\section{INTRODUCCIÓN}

El presente artículo plantea una propuesta de mejoramiento de los servicios en salud mental que se ofrecen desde el área de bienestar universitario en las instituciones de educación superior colombianas conforme a las falencias encontradas en el mismo. Inicialmente, se revisa la definición del concepto de bienestar universitario en la que se analizan algunos aspectos por mejorar en la oferta de servicios en salud mental y a partir de estos aspectos por mejorar se hace la propuesta.

El servicio de bienestar universitario que se brinda en las instituciones de educación superior en Colombia, ha sido definido en la Ley 30 de 1992, como el conjunto de actividades que se orientan al desarrollo físico, psico-afectivo, espiritual y social de los estudiantes, docentes y personal administrativo (Congreso de la Republica de Colombia 1992).

Teniendo en cuenta, que esta conceptualización quedaba incompleta, con la expedición del Decreto 1205 de 2010 (Ministerio de Educación Nacional, 20I0), se estableció en el artículo 6.6 que las acciones de bienestar universitario deben incluir: programas y servicios preventivos en salud, primeros auxilios o situaciones de riesgo, la organización de bolsas de empleo, estimular el desarrollo de aptitudes artísticas y la apreciación del arte; caracterizar la deserción y promover estrategias para disminuirla; $y$ en el caso de los programas a distancia o virtuales, plantear estrategias de participación de los estudiantes en los planes de bienestar universitario.

No obstante, en ninguna de las dos definiciones se deja en claro, qué servicios específicos en salud deben ofrecer las instituciones de educación superior y en qué modalidades; por ende, algunas universidades pueden ofrecer servicios de atención personalizada y talleres enfocados en abordar temas propios de la salud física, pero no necesariamente trabajar temas y necesidades en salud mental (Contecha, 2016; Contecha \& Jaramillo, 20II).

De igual manera, tampoco se menciona el tema de la cobertura de los servicios en salud para la comunidad estudiantil y de acciones que puedan implementarse para garantizarla, lo cual representa un problema relevante a considerar, ya que hay varias universidades que tienen entre 1000 y 25000 estudiantes, y el personal que labora en bienestar universitario no es suficiente' para dar una respuesta oportuna frente a estas gran cifra de personas (Ministerio de Educación, 2016; Contecha, 2016).

Desde esta perspectiva, es importante considerar a nivel legal la necesidad de que a futuro en Colombia se establezca una ley específica que defina a profundidad los servicios de bienestar universitario, y particularmente los servicios en salud, que no se quede una política gubernamental como en el caso del documento Lineamientos de Política de Bienestar para las Instituciones de Educación Superior (Ministerio de Educación, 2016) sino que adquiera un impacto a largo plazo.

Al respecto, sería adecuado que se hiciera una conceptualización diferencial entre servicios en salud física y servicios en salud mental. Los primeros se centran en prevenir $y$ atender enfermedades corporales desde disciplinas como la medicina, enfermería o fisioterapia, mientras que los segundos, se focalizan en atender problemas personales, familiares y de adaptación social, relacionados con hábitos de vida inadecuados y estados emocionales disfuncionales desde campos del saber cómo: la psicología, la psicopedagogía y el trabajo social (Martínez, Piqueras \& Ramos, 20I0).

Dentro de los hábitos de vida inadecuados más frecuentes están: una mala alimentación, el sedentarismo, fumar, ingerir bebidas alcohólicas con frecuencia, no dormir el número de horas requeridas por el cuerpo para descansar, la promiscuidad y la falta de una rutina de estudio establecida (Suescún-Carrero, Sandoval-Cuellar, Hernández-Piratoba, Araque-Sepúlveda, Fagua-Pacavita, Bernal-Orduz \& Corredor-Gamba, 2017; Sánchez-Ojeda \& De Luna-Bertos, 2015).

Por otro lado, como parte de los estados emocionales disfuncionales se encuentran: la ansiedad, depresión y la irritabilidad (Arrieta, Díaz \& González, 20I4) que pueden llegar a afectar la autoestima de los estudiantes y su deseo de vivir (Micin \& Bagladi, 20I I), así como la calidad de sus relaciones interpersonales (Chau \& Vilela, 2017; Pérez-Villalobos, Bonnefoy-Dibarrat, Cabrera-Flores, Peine-Grandón, Abarca, Baqueano-Rodríguez, Jiménez-Espinoza, 2012).

Estos problemas de la salud mental, cada vez están afectando a más estudiantes en las instituciones de educación superior colombianas (Marenco-Escuderos, Suarez-Coronado \& Palacio-Sañudo, J, 2017; Pinzón-Amado, 2017; Cardona-Arias, Pérez-Restrepo, Rivera-Ocampo, Gómez-Martínez \& Reyes, 2015; Arrieta, Díaz \& González, 2014; Vargas, Lemos, Toro \& Londoño, 2008; Palacio \& Martínez, 2007), quienes están ingresando más jóvenes a las universidades ${ }^{2}$ y son difíciles de abordar en su totalidad, por representar un elevado número de personas. Es por esto que los profesionales en psicología que trabajan en las áreas de bienestar universitario tienen el reto de redefinir su rol y la forma de ofertar sus servicios de atención a los estudiantes en salud mental (Vásquez, 20I2).

\section{DISCUSIÓN TEÓRICA}

El profesional en psicología que trabaje en bienestar universitario, debe brindar un servicio de consejería psicológica o counseling dirigido a los estudiantes, tanto a nivel personalizado como grupal que sea de buena calidad (Brunner, Wallace, Reymann, Sellers, McCabe, 20I4) es decir que promueva su bienestar mental y académico a la vez que garantice la cobertura de sus servicios (Scheel, Berman, Friedlander, Conoley, Duan, \& Whiston, 20I I; Pinilla, 2008).

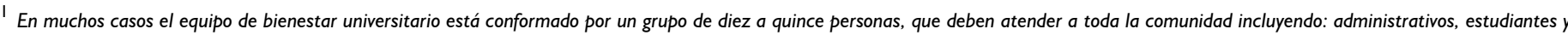
docentes.

${ }^{2}$ Hay estudiantes que están ingresando con edades que oscilan entre los 15 y 17 años de edad, por lo que aún no tienen la madurez requerida para enfrentar situaciones de riesgo por sí solos.
} 
El concepto de consejería o counseling, es más amplio que el de orientación escolar (Scheel, et al, 20ll; Ministerio de Educación Nacional, 1994) que suelen desempeñar los psicólogos escolares o los psicopedagogos, porque abarca todas aquellas acciones de evaluación, orientación y capacitación en temas de tipo educativo, hábitos de vida saludable y salud mental, que se pueden ofrecer a los educandos tanto de programas de pregrado presencial como virtuales y a distancia para que puedan tomar buenas decisiones en diferentes ámbitos de su vida y alcancen un estado de bienestar emocional.

Actualmente, los psicólogos de bienestar universitario ofrecen principalmente, sesiones de consejería psicológica y de acompañamiento, personalizadas a aquellos estudiantes con hábitos de vida inadecuados o problemas emocionales que solicitan el servicio o son remitidos por las facultades de cada universidad, y también desarrollan espacios de formación grupal, como campañas, talleres y conversatorios (Contecha, 2016; Montoya-Vásquez, Urrego-Velásquez \& Páez-Zapata, 2014; Pinilla, 2008).

Ambos servicios individuales como grupales son presenciales, y en ocasiones no logran dar cobertura a todos los estudiantes, por lo cual se requiere implementar cambios como ampliar la oferta de los servicios antes mencionados ya no a nivel institucional sino por programa académico y en modalidad tanto presencial como virtual.

En otros países, ya existen espacios de consejería psicológica virtual (Baker \& Mike, 20I I), como páginas web, videoconferencias y chats, mediados por las tecnologías de la información y de la comunicación como el computador - la tableta que tengan conexión a Internet (Martínez de Salvo, 2010; Efstathiou, 2009) en los que el psicólogo tiene la posibilidad de dialogar con el estudiante en tiempo real aunque estén en contextos diferentes y además puede facilitarle material de autoayuda como textos, videos, e imágenes que complementan los procesos que se desarrollen interactivamente (Richards \& Viganó, 2013).

Este tipo de consejería beneficiaria mucho a los estudiantes de educación virtual y a distancia, que viven lejos de las ciudades capitales o que no tienen tiempo de interactuar directamente con los psicólogos de bienestar universitario (GonzálezBrignardello, \& Sánchez-Elvira, 2016; Estévez, CastroMartínez, Rodríguez, 2015), ya que les daría la posibilidad de formular inquietudes que puedan tener a nivel académico $o$ en la interacción con los tutores, solicitar orientación o consejo frente a determinados temas de su vida personal e incluso pedir ayuda cuando estén viviendo una crisis emocional ( $\mathrm{Da}$ Silva, Siegmund \& Bredemeier, 2015; Vásquez \& Rodríguez, 2007; Speyer \& Zack, 2003).

Por ejemplo, si un estudiante de un programa de pregrado de educación virtual y a distancia, requiere orientación psicológica podría solicitarla a través de herramientas como el Chat (servicio de mensajería instantánea) o por correo electrónico, con el fin de agendar una sesión con el psicólogo de bienestar universitario virtual y explicarle el motivo de consulta.

Posteriormente, la sesión de evaluación inicial se podría efectuar mediante una videoconferencia, en la cual el estudiante y el psicólogo puedan interactuar visual y verbalmente para abordar el problema o necesidad. En otras sesiones subsecuentes, el psicólogo como parte del proceso de orientación e intervención tendría la opción de seguirlo haciendo de manera individualizada o también grupal, invitando a varios estudiantes con problemas similares a participar de un foro virtual, en el que pueden dialogar o se puede desarrollar un taller.

De igual forma, así como los estudiantes de educación virtual y a distancia se benefician de este servicio, también podrían hacerlo aquellos estudiantes que hacen parte de programas de pregrado presenciales que, por diversos motivos, como el cruce de su jornada de clases con el horario de atención diurna de bienestar no pueden acceder a los servicios presenciales, y tendrían la alternativa de hacerlo vía on-line en otros momentos del día (Mallen, Vogel \& Day, 2005; Ruiz, 2005).

Sin embargo, hay ciertos dilemas que existen en torno a la asesoría psicológica virtual que no se han resuelto aún (Poh, Rafidah \& Haslee, 2013; Rummell \& Joyce, 2010) en Colombia. Por ejemplo, habría que preguntarse qué criterios definirían un servicio de consejería psicológica virtual de buena calidad, qué regulaciones debería tener la prestación de este tipo de servicios, y qué alcances podría tener con miras a lograr una cobertura de toda la comunidad estudiantil.

A nivel de la calidad (García, Guerrero \& Granados, 2015; Baker \& Mike, 20II) un servicio de consejería psicológica virtual debe cumplir con ciertos criterios como son: I) informar a los estudiantes de la oferta de servicios a través de una página web de fácil acceso, que tenga contenidos claros y precisos; 2) definir la oferta de servicios con fundamento en preguntas relacionadas con las necesidades de los estudiantes ${ }^{4}$ ; 3) dar datos de contacto y horarios de atención de los profesionales que estén a cargo de los servicios de tal manera que los estudiantes les puedan contactar; 4) brindar material de autoayuda como lecturas o videos, relacionados con las preguntas y necesidades de los estudiantes; 5) plantear rutas de acción a manera de flujogramas, que orienten a los estudiantes en el manejo de crisis emocionales, ideación suicida y situaciones de emergencias (Da Silva, Siegmund \& Bredemeier, 2015); y 6) definir protocolos internos para abordar diferentes tipos de situaciones de tipo emocional y académico que puedan presentar los estudiantes.

En cuanto a la regulación de los procedimientos profesionales en consejería psicológica virtual, sería pertinente que el

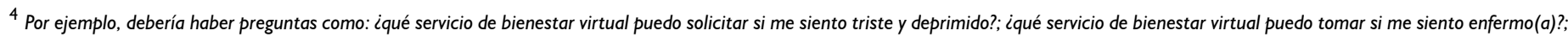

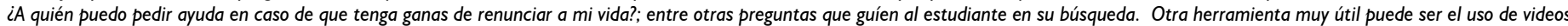
informativos o con testimonios de personas en los que se motive al estudiante a pedir ayuda y se le muestre como hacerlo.
} 
Colegio Colombiano de Psicólogos, incluyera dentro de la Ley 1090 de 2006 (Congreso de la República, 2006), conceptos como: evaluación psicológica virtual, procesos de intervención virtuales, y consejería psicológica virtual (Baker \& Mike, 20I I), de tal manera que éstos se puedan comenzar a diferenciar de las prácticas psicológicas presenciales, y se puedan reglamentar en diversos contextos (Lau, Mohd, \& Sharil, 20I3).

La idea de esta reglamentación es garantizar una buena calidad de los servicios virtuales, en términos de: la generación de una relación contra-actual entre el psicólogo y sus estudiantes a cargo en espacios virtuales; el manejo confidencial de la información; la generación de registros, formatos y de informes que permitan dar cuenta de los servicios prestados; y la evaluación de la satisfacción de los educandos conforme a los servicios prestados por el psicólogo (Lau, et al, 20I3).

El hecho de que se reglamenten este tipo de procedimientos ayudaría a minimizar la informalidad e improvisación en las interacciones que tenga el psicólogo con la comunidad estudiantil; y ayudaría a estandarizar sus acciones conforme a metas de trabajo específicas que den respuesta a las necesidades de éstos.

Por otra parte, en lo concerniente a los alcances de los servicios en consejería psicológica virtual, los psicólogos de bienestar universitario deben generar consciencia entre las directivas de las instituciones de educación superior en las que trabajan, para que se designe por cada programa académico de pregrado uno o dos psicólogos que desarrollen la oferta de servicios en consejería psicológica virtual y presencial (Haberstroh, Duffey, Evans, Gee, \& Trepal, 2007), de tal forma que se pueda ampliar la cobertura y se reduzca el impacto de los problemas de salud mental, conductas de riesgo y el deseo de desertar en los estudiantes (Estévez, Castro-Martínez \& Rodríguez, 2015), en la medida en que se pueda hacer un mejor seguimiento de los casos que requieren mayor atención, y de las necesidades particulares de los estudiantes (Sánchez, 2016; Suarez-Montes \& Díaz-Subieta, 2015; Mishna, Bogo \& Leigh, 20I3).

Universidades extranjeras que ofrecen servicios de consejería psicológica virtual

A continuación, se describen ejemplos de avances en consejería psicológica virtual que ya existen en otros países y pueden constituirse en modelos a seguir en Colombia.

En ciertos países del hemisferio norte, se ofrecen servicios de consejería psicológica o counseling a los estudiantes de pregrado que ingresan a las universidades, y tienen páginas web en las que se describe dicho servicio.

Tal es el caso de instituciones educativas inglesas como la Universidad de Cambridge, la Universidad de Oxford, la Universidad de Nothingham y la Universidad de Sussex, de instituciones canadienses como el Douglas College, el Sheridan College o el Confederation College y en Estados Unidos, la Universidad de lowa, la Universidad Estatal de Worcester y la Universidad de Memphis.
Al comparar las páginas web de estas instituciones, se encuentran en todas ciertos elementos en común como son: I) una breve definición del servicio de consejería psicológica o counseling; 2) la oferta de recursos de autoayuda como lecturas acerca de temas de interés; 3 ) directrices puntuales para los estudiantes en las que se les indica cómo acceder al servicio, horarios, lugares de atención, teléfonos y datos de profesionales; 4) información de actividades de apoyo y de orientación tanto individuales como grupales; y 5) teléfonos de contacto para manejar situaciones de emergencias.

Pese a que en ninguna página web se ofrecen servicios de consejería virtual como tal, las páginas web se pueden considerar una forma de consejería virtual en sí misma, debido a que informan al estudiante y en esa medida le orientan para la toma de decisiones en el manejo de problemas personales, mientras que en el caso de las instituciones de educación superior colombianas se ofrece información general en sus páginas web sobre servicios deportivos, culturales, de becas y en ciertos casos de salud, pero hace falta información específica que oriente a los estudiantes para manejar situaciones de crisis o problemas personales, lo cual es muy relevante, ya que los estudiantes pueden llegar a requerirlo en cualquier momento (Da Silva, Siegmund \& Bredemeier, 20I5).

Además, la mayoría de los servicios que se ofrecen desde bienestar universitario en las instituciones de educación superior colombianas, la mayoría se han enfocado principalmente en atender las necesidades de los estudiantes de educación presencial, pero tienden a dejar de lado las necesidades de los educandos de educación virtual y a distancia, a excepción de algunas universidades como la Universidad Nacional Abierta y a Distancia (Perdomo \& Cañón, 20 I I), la Universidad Santiago de Cali y Uniminuto Virtual y a Distancia UVD, así como otras en las cuales se dan herramientas básicas de ayuda a los estudiantes de estas modalidades.

\section{PROPUESTA DE MEJORAMIENTO}

Las universidades deben comenzar a pensar en la necesidad de que se oferten servicios de consejería psicológica en línea personalizados relacionados con (Janetius, 2016; Guevara, 2008): I) manejo de situaciones de crisis o depresión; 2) ansiedad académica; 3) bajo rendimiento académico; 4) gestión adecuada del tiempo y del dinero; 5) manejo de problemas familiares o personales que puedan interferir con temas académicos del educando; 6 ) acceso a becas y crédito como alternativas para evitar la deserción; y 7) abordaje de situaciones de emergencias tanto dentro como fuera de las instituciones.

Tales servicios podrían ofertarse mediante correo electrónico, mensajería instantánea, videoconferencias personalizadas (Chester \& Glass, 2006), videoconferencias masivas (tipo capacitaciones), videos pregrabados que aborden temas específicos; lecturas de apoyo o autoayuda, y vínculos virtuales con entidades externas como la línea de emergencias (I23), la policía, las clínicas, entre otros.

Otro aspecto relevante del tema, es que debería darse un 
servicio adicional de seguimiento virtual a estudiantes que hayan sido reportados en riesgo, ya sea por deserción académica o problemas personales, en el cual se pueda brindar un espacio de ayuda y orientación para estas personas (Donoso, Neira, \& Donoso, 2018; Escanés, Herrero, Merlino \& Ayllón, 2014).

Por ejemplo, Richards (2009) ha encontrado que los estudiantes que han tomado servicios de consejería psicológica virtual en el Trinity College de Dublin, Irlanda han mostrado altos niveles de satisfacción con el mismo y Wong, Bonn, Tam \& Wong (2018) también han encontrado lo mismo en estudiantes de la Universidad de Malasia. Esto demuestra que la modalidad de consejería psicológica virtual, puede tener un impacto positivo en los estudiantes si se fomenta una cultura de la interacción virtual (Carmona \& Rodríguez, 2017; Vásquez \& Arango, 20l2) en las instituciones de educación superior y si se definen buenos programas de capacitación ${ }^{5}$ (Pipoly, 2013; Ekong, 2006) y atención en consejería psicológica virtual (Poh, et al, 20l3).

Algunos beneficios derivados de la consejería psicológica virtual son la detección y atención oportuna de estudiantes con problemas personales o académicos con riesgo de desertar de sus estudios, el mejoramiento del proceso de seguimiento virtual a los estudiantes a lo largo de sus estudios, la ampliación en la cobertura del servicio de consejería psicológica tanto en términos del número de horas diarias como de la cantidad de estudiantes que acceden al mismo, y una mayor efectividad en la atención que se puede brindar a las solicitudes de los estudiantes.

\section{CONCLUSIONES}

Finalmente, y tomando en consideración los planteamientos previos, los profesionales que trabajan en bienestar universitario, el Ministerio de Educación Nacional y el Colegio Colombiano de Psicólogos deben reflexionar respecto a temas aún inconclusos como: la conceptualización sobre el tema de la consejería psicológica virtual en Colombia y su diferencia con la consejería psicológica presencial, la regulación de las prácticas de evaluación e intervención psicológica virtuales en el país; y actividades de capacitación al interior de las instituciones educativas para los psicólogos en esta nueva modalidad de trabajo que se está extendiendo progresivamente, de tal manera que puedan diseñar páginas web y materiales virtuales de uso accesible para los estudiantes (Castro, 2018).

Una forma de lograr dar respuesta a estos temas podría ser consultar con profesionales expertos en consejería procedentes de otros países, que compartan sus experiencias con profesionales locales, así como las buenas prácticas profesionales y las últimas tendencias en prácticas consejería virtual, con miras a que se pueda desarrollar el área de consejería psicológica en Colombia a nivel académico y profesional.
Dentro de las buenas prácticas profesionales en consejería psicológica virtual deberían tenerse en consideraciones algunas como: demostrar manejo de las plataformas informáticas y las herramientas que ofrecen para establecer contacto con los estudiantes; dar a conocer el servicio de consejería psicológica virtual a toda la comunidad universitaria virtual, y sensibilizarla frente al mismo; hacer seguimiento de los reportes de docentes y estudiantes para detectar oportunamente casos que requieran ayuda; usar el chat y el correo electrónico solo como herramientas de contacto inicial y la videoconferencia como herramienta de interacción con los estudiantes; explicar a los estudiantes la importancia de crear un ambiente de confidencialidad durante las interacciones (Iza, 20I8) y pedirles que diligencien formatos de consentimiento informado virtual para tener su aprobación ante cualquier procedimiento profesional que se vaya a efectuar; plantearle a los estudiantes las limitaciones del servicio, para clarificar así los alcances del mismo a nivel de los horarios de atención y del rol del psicólogo de bienestar; hacer un seguimiento constante de aquellos casos que se han atendido virtualmente; hacer un cierre de los casos atendidos solo hasta cuando finalicen su plan de estudios, para garantizar así una cobertura adecuada del servicio; y llevar un registro virtual del historial de cada estudiante atendido, que sea mantenido en reserva por los psicólogos de bienestar y sirva de sustento al trabajo realizado.

\footnotetext{
${ }^{5}$ Por ejemplo, a futuro se podrían desarrollar postgrados y cursos de capacitación en consejería psicológica virtual, que brinden herramientas a los psicólogos en esta área.
} 


\section{Referencias bibliográficas}

Arrieta, K; Díaz, S \& González, F. (2014). Síntomas de depresión y ansiedad en jóvenes universitarios: prevalencia y factores relacionados. Revista Clínica de Medicina de Familia, 7(I), 14-22. DOI: http://dx.doi.org/I0.432I/SI699-695X20I4000I00003. Artículo recuperado de: http://scielo.isciii.es/pdf/albacete/v7nl/original2.pdf

Baker, K \& Mike, R. (20II). Online counseling: The good, the bad, and the possibilities. Counseling Psychology Quarterly, 24(4), 34I-346. https://doi.org/I0.1080/095I5070.20II.632875

Brunner, J; Wallace, D; Reymann, L; Sellers, J \& McCabe, A. (2014). College counseling today: contemporary students and how counseling centers meet their needs. Journal of College Student Psychotherapy, 28, 257-324. DOI: https://doi.org/10.1080/875 68225.2014.948770. Artículo recuperado de: https://www.researchgate.net/publication/28327I539_College_Counseling_Today_ Contemporary_Students_and_How_Counseling_Centers_Meet_Their_Needs/download

Cardona-Arias, J; Perez-Restrepo, D; Rivera-Ocampo, S; Gómez-Martínez, J \& Reyes, A. (2015). Prevalencia de ansiedad en estudiantes univrsitarios. Revista Diversitas -Perspectivas en Psicología, I I(I), 79-89. ISSN: 1794-9998. Artículo recuperado de: http://www.scielo.org.co/pdf/dpp/vl Inl/vl Inla06.pdf

Carmona, E \& Rodríguez, E. (2017). Buenas prácticas en la educación superior virtual a partir de especificaciones de es-tándares eLearning. Sophia, I3(I), I3-26. DOI: http://dx.doi.org/I0.18634/sophiaj. I 3v. I i.345. Artículo recuperado de: http://www.scielo.org. $\mathrm{co} / \mathrm{pdf} / \mathrm{sph} / \mathrm{vl} 3 \mathrm{nl} / \mathrm{vl} 3 \mathrm{nla03}$.pdf

Castro, R. (20I8). Manejo de tecnología e información científica en la formación universitaria. Inclusión y Desarrollo, 2(5), 6I-76. DOI: http://dx.doi.org/I0.26620/uniminuto.inclusion.5.2.2018.63-82. Artículo recuperado de: http://revistas.uniminuto.edu/index. $\mathrm{php} / \mathrm{YYD} /$ article/view/I796/1697

Chau, C \& Vilela, P. (2017). Determinantes de la salud mental en estudiantes universitarios de Lima y Huánaco. Revista de Psicología, 35(2), 387-422. DOI: http://dx.doi.org/I0.18800/psico.201702.00I. Artículo recuperado de: http://www.scielo.org.pe/pdf/psico/ v35n2/a0 Iv35n2.pdf

Chester, A \& Glass, C. (2006). Online counseling: a descriptive analysis of therapy services on the Internet. British Journal of Guidance \& Counseling, 34(2), I45-160. DOI: https://doi.org/I0.1080/03069880600583 I70. Artículo recuperado de: https://www.researchgate. net/publication/2475 17087 Online_counselling_A_descriptive_analysis_of_therapy_services_on_the_Internet/download

Congreso de la República de Colombia. (2006). Ley 1090 de 2006. Por la cual se reglamenta el ejercicio de la profesión de Psicología, se dicta el Código Deontológico y Bioético y otras disposiciones. Documento recuperado de: http://colpsic.org.co/aym_image/files/ LEY_1090_DE_2006.pdf

Congreso de la República de Colombia. (1992). Ley 30 de Diciembre 28 de 1992 por el cual se organiza el servicio público de la Educación Superior. Documento recuperado de: https://www.mineducacion.gov.co//62 I/articles-86437_Archivo_pdf.pdf

Contecha, L. (2016). Un estado del arte del bienestar universitario en Colombia. Revista Perspectivas Educativas, 109-127. Artículo recuperado de: http://revistas.ut.edu.co/index.php/perspectivasedu/article/download/789/617.

Contecha, L \& Jaramillo, L. (20I I). El bienestar universitario subordinado a una modernidad instrumental. Revista U.D.C.A Actualidad \& Divulgación Científica, I4(I), I0I-I09. ISSN: 0I23-42-26. Artículo recuperado de: http://www.scielo.org.co/pdf/rudca/vl4nl/ v|4nlal3.pdf

Da Silva, J; Siegmund, G \& Bredemeier, J. (2015). Crisis interventions in online psychological counseling. Trends in Psychiatry \& Psychotherapy, 37(4), I7I-I82. DOI: http://dx.doi.org//0.1590/2237-6089-2014-0026. Artículo recuperado de: http://www.scielo. br/scielo.php?script $=$ sci_arttext\&pid=S2237-608920I5003400I $7 \mathrm{I}$

Donoso, S; Neira, T \& Donoso, G. (2018). Sistemas de Alerta Temprana para estudiantes en riesgo de abandono de la Educación Superior. Ensaio: aval. pol. públ. Educ, 26(100), 944-967. DOI: https://doi.org/10.1590/S0I04-403620I800260l494. Artículo recuperado de: https://www.researchgate.net/publication/326984969_Sistemas_de_Alerta_Temprana_para_estudiantes_en_ riesgo_de_abandono_de_la_Educacion_Superior

Efstathiou, G. (2009). Students' psychological web consulting: function and outcome evaluation. British Journal of Guidance \& Counseling, 37(3), 243-255. DOI: https://doi.org/I0.1080/03069880902956983. 
Ekong, J. (2006). What Factors Facilitate Online Counselor Training?. Experiences of Campus Alberta Graduate Students. Journal of Distance Education, 2 I (I), I-I4. ISSN-0830-0445. Artículo recuperado de: https://files.eric.ed.gov/fulltext/EJ807807.pdf

Escanés, G; Herrero, V; Merlino, A \& Ayllón, S. (2014). Deserción en educación a distancia: factores asociados a la elección de modalidad como desencadenantes del abandono universitario. Virtualidad, Educación y Ciencia, 5(9), 45-55. ISSN: I853-6530. Artículo recuperado de: https://revistas.unc.edu.ar/index.php/vesc/article/view/9549

Estévez, J; Castro-Martínez, J; Rodríguez, H. (20I5). La educación virtual en Colombia: exposición de modelos de deserción. Apertura, 7(I), I-I0. ISSN: 2007-1094. Artículo recuperado de: http://www.udgvirtual.udg.mx/apertura/index.php/apertura/article/view/583

García, A; Guerrero, R \& Granados, J. (2015). Buenas prácticas en los entornos virtuales de enseñanza-aprendizaje. Revista Cubana de Educación Superior, 3, 76-88. ISSN: 0257-43I4. Artículo recuperado de: http://scielo.sld.cu/pdf/rces/v34n3/rces063 I5.pdf

González-Brignardello, M \& Sánchez-Elvira, A. (2016). Prevention of student dropout in higher distance education: Positive Technology. The Online, Open and Flexible Higher Education Conference 2016 - Proceedings. 843-856. Artículo recuperado de: https://www. researchgate.net/publication/309538665_Prevention_of_student_dropout_in_higher_distance_education_Positive_Technology

Guevara, M. (2008). Consultorio psicológico virtual: Una alternativa preventiva para la psicología. Av. Psicol, I6(I), 53-68. Artículo recuperado de: http://www.unife.edu.pe/pub/revpsicologia/consultoriopsicologico.pdf

Haberstroh, S; Duffey, T; Evans, M; Gee, R \& Trepal, H. (2007). The Experience of Online Counseling. Journal of Mental Health Counseling, 29(3), 269-282. DOI: I0.17744/mehc.29.3.j34465 I 26 I w357v2. Artículo recuperado de: https://www.researchgate.net/ publication/228674249_The_Experience_of_Online_Counseling

Iza, D. (20I8). Estudio de casos de la relación terapéutica en una intervención realizada por videoconferencia. Maestría Thesis. Bogotá, D.C: Universidad Nacional de Colombia. Documento recuperado de: http://bdigital.unal.edu.co/70447/2/Daniellza.2018. pdf

Janetius, S. (2016). Evidence-Based Mental Health Counseling Model for College Students. The International Journal of Indian Psychology, 4(I), I67-I76. DOI: https://doi.org/I0.1080/875682208019522/4. Artículo recuperado de: https://www.researchgate. net/publication/309705074_Mental_Health_Counselling_Model_for_College_Students/download

Lau, P; Mohd, R \& Sharil Lim, H. (2013). Understanding the Two Sides of Online Counseling and their Ethical and Legal Ramifications. Procedia - Social and Behavioral Sciences, 103, I 243 - I25I. https://doi.org/10.1016/j.sbspro.2013. I0.453. Artículo recuperado de: https://www.researchgate.net/publication/275538676_Understanding_the_Two_Sides_of_Online_Counseling_and_their_Ethical_ and_Legal_Ramifications/fulltext/554b4bcd0cf29752ee7c54f0/275538676_Ūnderstanding_the_Two_Sides_of_Online_Counseling_ and_their_Ethical_and_Legal_Ramifications.pdf?origin=publication_detail

Mallen, M; Vogel, D \& Day, S. (2005). Online counseling: reviewing the literature from a counseling psychology framework. The Counseling Psychologist, 33(6), 819-87I. DOI: 10.1 I77/00I 1000005278624. Artículo recuperado de: https:/www.researchgate. net/profile/David_Vogel3/publication/238078522_Online_Counseling_Reviewing_the_Literature_From_a_Counseling_Psychology_ Framework/links/02e7e5 I c483ea37d7a000000.pdf

Marenco-Escuderos, A., Suárez-Colorado, Y., \& Palacio-Sañudo, J. (2017). Burnout académico y síntomas relacionados con problemas de salud mental en universitarios colombianos. Psychologia: Avances de la Disciplina, II(2), 45-55. ISSN I900-2386. Artículo recuperado de: https://revistas.usb.edu.co/index.php/Psychologia/article/view/2926/2756

Martínez, A; Piqueras, J \& Ramos, V. (2010). Inteligencia emocional en la salud física y mental. Electronic Journal of Research in Educational Psychology, 8(2), 86 I-890. E-ISSN: I696-2095. Artículo recuperado de: http://www.redalyc.org/pdf/293 I/293 I 22002020. pdf

Martínez de Salvo, F. (20I0). Herramientas de la Web 2.0 para el aprendizaje 2.0. Revista de Artes y Humanidades UNICA, I I(3), I74-190. ISSN: I 3 I7-I02X. Artículo recuperado de: https://www.redalyc.org/pdf/I 70I/I 70121969008.pdf

Micin, S \& Bagladi, V. (20II). Salud mental en estudiantes universitarios: Incidencia de psicopatología y antecedentes de conducta suicida en población que acude a un servicio de salud estudiantil. Terapia Psicológica, 29(I), 53-64. ISSN 07I8-4808. Artículo recuperado de: https://scielo.conicyt.cl/pdf/terpsicol/v29n I/art06.pdf

Ministerio de Educación Nacional. (2016). Lineamientos de Política de Bienestar para las Instituciones de Educación Superior. Documento recuperado de: https://www.mineducacion.gov.co/I 759/articles-3603 |4_recurso.pdf 
Ministerio de Educación Nacional. (2010). Decreto 1295 del 20 de abril de 20I0. Por el cual se reglamenta el registro calificado de que trata la Ley 1188 de 2008 y la oferta y desarrollo de programas académicos de educación superior. Documento recuperado de: https://www.mineducacion.gov.co/I621/articles-229430_archivo_pdf_decreto I 295.pdf

Ministerio de Educación Nacional de Colombia. (1994). Decreto 1860 de Agosto 3 de 1994. Por el cual se reglamenta parcialmente la Ley II 5 de 1994, en los aspectos pedagógicos y organizativos generales. Documento recuperado de: https://www.mineducacion. gov.co/I621/articles-86240_archivo_pdf.pdf

Mishna, F; Bogo, M \& Leigh, J. (2013). Cyber Counseling: Illuminating Benefits and Challenges. Clinical Social Work Journal. Artículo recuperado de: DOI I0.1007/s I06I5-013-0470-I.

Montoya-Vásquez D; Urrego-Velásquez D \& Páez-Zapata, E. (20l4). Experiencia en la coordinación de programas de bienestar universitario: la tensión entre el asistencialismo y el desarrollo humano de los estudiantes. Rev. Fac. Nac. Salud Pública, 32(3), 355363. ISSN: 0I20-386X. Artículo recuperado de: http://www.scielo.org.co/pdf/rfnsp/v32n3/v32n3al0.pdf

Palacio, J \& Martínez, Y. (2007). Relación del rendimiento académico con la salud mental en jóvenes universitarios. Psicogente, I0(I8), I I3-128. Artículo recuperado de: https://dialnet.unirioja.es/descarga/articulo/6 I 13749.pdf

Pérez-Villalobos, C; Bonnefoy-Dibarrat, C; Cabrera-Flores, A; Peine-Grandón, S; Abarca, K; Baqueano-Rodríguez, M; JiménezEspinoza, J. (2012). Problemas de salud mental en alumnos universitarios de primer año de Concepción, Chile. Anales de Psicología, 28(3), 797-804. DOI: http://dx.doi.org/10.60I8/analesps.28.3.15607I. Artículo recuperado de: http://www.redalyc.org/ pdf/I67/167237740I8.pdf

Perdomo, M \& Cañón, V. (20I I). Bienestar universitario virtual, una estrategia para el fortalecimiento de la comunidad unadista con el uso de las TICs. Revista de Investigaciones UNAD Bogotá, I0(2), 109-I25. DOI: https://doi.org/I0.22490/2539/887.757. Artículo recuperado de: https://academia.unad.edu.co/images/investigacion/hemeroteca/revistainvestigaciones/volumen I Onum $2 \% 20$ 201 I/7.\%20Bienestar\%20universitario\%20virtual.pdf

Pinilla, M. (2008). El cuidado de lo humano en el contexto universitario. Aportes de un equipo de trabajo. Primera Edición. Bogotá, D.C: Editorial Pontificia Universidad Javeriana. ISBN: 9789587I6I496.

Pinzón-Amado, A. (2017). Prevalencia de trastornos mentales y consumo de sustancias psicoactivas en estudiantes universitarios. Revista de la Universidad Industrial de Santander Salud, 49(I). Artículo recuperado de: https://revistas.uis.edu.co/public/journals/2/ docs/prevalencia_de_trastornos_mentales.pdf

Pipoly, L. (2013). Counselor Education Curriculum and Online Counseling. Journal of Instructional Research, 2, 48-57. Artículo recuperado de: https://files.eric.ed.gov/fulltext/EJI I27652.pdf

Poh, L; Rafidah, J \& Haslee, A. (2013). Understanding the two sides of online counseling and their ethical and legal ramifications. Procedia - Social and Behavioral Sciences, 103, 1243 - I25I. DOI: https://doi.org/10.1016/j.sbspro.2013.10.453. Artículo recuperado de: https://ac.els-cdn.com/SI8770428I3038986/I-s2.0-SI8770428I3038986-main.pdf?_tid=e50fb9f2-9044-490a-8e4b-7e5928988 cce\&acdnat $=|550674| 6 \mid \_64$ ec9347e36cdba76e7a59e576a89240

Richards, D \& Viganó, N. (2013). Online Counseling: A Narrative and Critical Review of the Literature. Journal of Clinical Psychology, 69(9), 994-I0I I. DOI: 10.1002/jclp.2 1974. Artículo recuperado de: https://www.researchgate.net/publication/23658I528_Online_ Counseling_A_Narrative_and_Critical_Review_of_the_Literature

Richards, D. (2009). Features and benefits of online counseling: Trinity College online mental health community. British Journal of Guidance \& Counseling, 37(3), 23I - 242. DOI: 10.1080/03069880902956975. Artículo recuperado de: https://www.google.com.co/h?q=Features + and + benefits + of +online + counseling\% $3 \mathrm{~A}+\mathrm{Tr}$ inity + College + online + mental + health + community\&oq $=$ Features + and + benefits + of + online + counseling$\% 3 \mathrm{~A}+$ Trinity + College + online + mental + health + community\&aqs = chrome. $69 \mathrm{i} 57.1010 \mathrm{j} 0 \mathrm{j} 7 \&$ sourceid $=$ chrome\&ie $=$ UTF-8

Ruiz, F. (2005). Claves para la comunicación: la intervención psicológica a través de Internet. Comunicar, 24, I88-191. ISSN: II343478. Artículo recuperado de: https://www.revistacomunicar.com/indice/articulo.php?numero=24-2005-28

Rummell, C \& Joyce, A. (2010). 'So wat do u want to wrk on 2day?': The Ethical Implications of Online Counseling. Ethics \& Behavior, 20(6), 482-496. DOI: https://doi.org/I0.1080/10508422.2010.52I450

Sánchez, G. (2016). Bienestar en redes sociales. Su utilidad en educación virtual y a distancia. PSIENCIA - Revista Latinoamericana de Ciencia Psicológica, (8)2, I-13. DOI: 10.5872/psiencia/8.2.22. Artículo recuperado de: https://www.redalyc.org/ 
Sánchez-Ojeda, M \& De Luna-Bertos, E. (2015). Hábitos de vida saludable en la población universitaria. Revista Nutrición Hospitalaria, 3I(5), I9I0-1919. DOI: 10.3305/nh.2015.31.5.8608. Artículo recuperado de: http://scielo.isciii.es/pdf/nh/v3 In5/03revision03.pdf

Scheel, M; Berman, M; Friedlander, M; Conoley, C; Duan, C \& Whiston, S. (20I I). Whatever Happened to Counseling in Counseling Psychology?. The Counseling Psychologist 20(10), I-20. DOI: I0.I I77/00 I 1000010380278 . Artículo recuperado de: https://www. apa.org/education/ce/counseling-in-counseling.pdf

Speyer, C \& Zack, J. (2003). Online counseling: beyond the pros \& cons. Artículo recuperado de: https://www.researchgate.net/ publication/2655656/8

Suarez-Montes, N \& Díaz-Subieta, L. (20I5). Estrés académico, deserción y estrategias de retención de estudiantes en la educación superior. Revista Salud Pública, I7(2), 300-313. DOI: http://dx.doi.org/10.I5446/rsap.v17n2.5289I. Artículo recuperado de: http:// www.scielo.org.co/pdf/rsap/v17n2/v17n2al3.pdf

Suescún-Carrero, S; Sandoval-Cuellar, C; Hernández-Piratoba, F; Araque-Sepúlveda, I; Fagua-Pacavita, L; Bernal-Orduz, F \& Corredor-Gamba. (20I7). Estilos de vida en estudiantes de una universidad de Boyacá, Colombia. Rev. Fac. Med, 65(2), 227-23I. DOI: https://doi.org/10.I5446/revfacmed.v65n2.58640. Artículo recuperado de: http://www.scielo.org.co/pdf/rfmun/v65n2/0I2000 I I-rfmun-65-02-227.pdf

Vargas, O; Lemos, M; Toro, B \& Londoño, N. (2008). Prevalencia de trastornos mentales en estudiantes universitarios usuarios del servicio de psicología. International Journal of Psychological Research, I (2), 2 I-30. ISSN: 20 I I-2084. Artículo recuperado de: http:// bdigital.ces.edu.co:8080/repositorio/bitstream/10946/3753/I/I.pdf

Vásquez, J. (2012). Reflexión sobre cómo los psicólogos perciben las intervenciones psicológicas online. Revista Virtual Universidad Católica del Norte, 35, 346-365. ISSN: 0124-582 I. Artículo recuperado de: http://revistavirtual.ucn.edu.co/index.php/RevistaUCN/ article/view/365

Vásquez, C \& Arango, S. (20I2). Estrategias de participación e interacción en entornos virtuales de aprendizaje. Anagramas, I0(20), 95-I08. DOI: https://doi.org/I0.22395/angr.vIOn20a6. Artículo recuperado de: http://www.scielo.org.co/pdf/angr/vI0n20/ v10n20a07.pdf

Vásquez, C \& Rodríguez, M. (2007). La deserción estudiantil en educación superior a distancia: perspectiva teórica y factores de incidencia. Revista Latinoamericana de Estudios Educativos, 37(3-4), I07-I22. ISSN: 0185-I284. Artículo recuperado de: http:// www.alfaguia.org/alfaguia/files/I342815884_I494.pdf

Wong, K; Bonn, G; Tam, C \& Wong, C. (2018). Preferences for online and/or face-to-face counseling among University students in Malasia. Frontiers in Psychology, 9(64), I-5. DOI: 10.3389/fpsyg.2018.00064. Artículo recuperado de: https://www.frontiersin.org/ articles/10.3389/fpsyg.2018.00064/full 